oesophageal balloon distention. During fMRI, 480 T2* weighted images per slice $(40 \times 3 \mathrm{~mm}$ slices, 0.3 interslice gap, TE $30 \mathrm{~ms}$, TR $2500 \mathrm{~ms}$, flip angle $80^{\circ}$, matrix size 642, sum of images per scan $=19,200)$ were captured to illustrate blood oxygen level dependent (BOLD) contrast during the different experimental events. The effects of extraversion on fMRI response during these events were subsequently determined using ANOVA brain activation mapping analyses within XBAM, a statistical package of image processing and statistical inference.

Results There was a diversity of extraversion scores (range 622), which did not influence pain threshold or rating. High extraversion was associated with significantly greater activity in the left cuneus (Brodmann Area (BA) 18) during rest ( $\mathrm{p}<$ $0.001)$ and the right insula (BA13) during both anticipation ( $\mathrm{p}<$ $0.0002)$ and pain $(p<0.0008)$. Low extraversion was associated with significantly greater brain activity in numerous regions during pain anticipation, including the bilateral precuneus (BA31), bilateral lingual gyrus (BA18) and the right inferior temporal gyrus $(\mathrm{p}<0.0001)$.

Conclusion Our results suggest that the brain processing of pain is influenced by the personality dimension of extraversion and therefore like other personality dimensions such as neuroticism, extraversion should be controlled for in brain imaging studies of pain in health and disease.

Disclosure of Interest None Declared.

\section{OC-067 ENHANCED PERCEPTION OF PROXIMAL GASTRO-OESOPHAGEAL REFLUX: IMPAIRED MUCOSAL INTEGRITY OR DISTINCT SENSORY INNERVATION?}

P Woodland* , C Lee, R Aktar, E Mthunzi, LA Blackshaw, SL Preston, D Sifrim. Barts and the London School of Medicine and Dentistry, Queen Mary University of London, London, UK

\subsection{6/gutjnl-2014-307263.67}

Introduction In patients with GORD, including refractory disease, reflux events reaching the proximal oesophagus are more likely to be perceived than those only reaching the distal oesophagus. There is also experimental data suggesting an increased sensitivity of the proximal oesophagus relative to the distal. As such, the proximal oesophagus is likely to be highly significant in the pathogenesis of GORD symptoms. Reasons for this proximal oesophageal sensitivity are not clear, but may include reflux volume, impairment in mucosal integrity or changes in sensory innervation. It has recently been shown that distal mucosal integrity (its ability to perform a protective barrier function) is more vulnerable to acid exposure in GORD than in controls. The integrity of the proximal oesophagus has not been tested. To our knowledge, there are no studies evaluating mucosal afferent innervation of the distal and proximal oesophagus. We aimed to compare mucosal integrity and afferent nerve distribution in the proximal and distal oesophagus in patients with heartburn without oesophagitis.

Methods In 23 patients with heartburn and 10 healthy volunteers baseline proximal and distal oesophageal impedance was measured in vivo. Oesophageal mucosal biopsies from the distal and proximal oesophagus were taken and baseline transepithelial electrical resistance (TER) was measured in Ussing chambers. Biopsies were examined immunohistochemically for presence and location of calcitonin gene-related peptide (CGRP) immunoreactive nerve fibres.

Results Baseline impedance was higher in the proximal than in the distal oesophagus in healthy volunteers $(2935 \pm 204 \Omega$ vs. $2234 \pm 290 \Omega, \mathrm{p}<0.05)$ and in patients $(2949 \pm 183 \Omega$ vs.1945 $\pm 235 \Omega, p<0.001)$. However, baseline TER was not significantly different between proximal and distal oesophagus, or between patients with heartburn and healthy volunteers. Mucosal CGRP-immunoreactive nerves were located more superficially in the proximal oesophagus compared to the distal oesophagus in healthy controls $(12.3 \pm 0.9$ vs. 23.8 \pm 1.2 cells from lumen, $\mathrm{p}<0.001)$ and in patients $(5.7 \pm$ 0.7 vs. $22.2 \pm 2.7$ cells from lumen, $\mathrm{p}<0.0001)$. Moreover, these nerves were located closer to the lumen in patients with heartburn compared to asymptomatic controls $(5.7 \pm 0.7$ vs. $12.3 \pm 0.9, \mathrm{p}<0.001)$.

Conclusion The baseline mucosal integrity of the proximal oesophagus is not more impaired than that of the distal, nor is it more impaired in patients with heartburn symptoms versus healthy controls.

Increased sensitivity of the proximal oesophagus in GORD may instead be associated with a more superficial location of mucosal afferent nerves. Topical protection of the proximal oesophageal mucosa is a potential treatment strategy to reduce this sensitivity.

Disclosure of Interest None Declared.

\section{OC-068 THE IMPACT OF ENDOSCOPIC THERAPY ON PATIENT-PERCEIVED OUTCOME AND QUALITY OF LIFE IN SPHINCTER OF ODDI DYSFUNCTION}

B Paranandi*, VTF Cheung, D Joshi, GH El-Sayed, GJ Johnson, SP Pereira, GJ Webster, MH Chapman. Pancreaticobiliary Medicine, University College London Hospitals, London, UK

\subsection{6/gutjnl-2014-307263.68}

Introduction Biliary Sphincter of Oddi dysfunction (SOD) is a benign but often debilitating condition. Significant improvement in pain following endoscopic sphincterotomy or sphincteroplasty (ES) in patients with Type 1 SOD, is excellent. Symptomatic improvement in patients with type 2 or 3 SOD is less favourable (reported $50-70 \%$ and $30-50 \%$ respectively). We aim to determine the impact of ES, on pain symptoms and global quality of life (QOL) in these groups, which has not previously been well defined.

Methods An ERCP database and electronic clinic lists (from September 2011 to 2013) were analysed to identify all cases of suspected SOD. Patients underwent a telephone questionnaire. The Glasgow Benefit Inventory (GBI), which assesses multiple physical, emotional and social parameters, was used to quantify global post-interventional QOL benefit. Total GBI scores can range from -100 (maximal negative benefit) to +100 (maximal positive benefit).

Results 163 new patients with suspected biliary SOD were identified of whom 89 underwent ERCP. 3 patients were excluded due to an alternative diagnosis at ERCP. The remaining cohort was predominantly Female (87\%) and

\begin{tabular}{llll} 
Abstract OC-068 Table 1 & \multicolumn{3}{c}{ Median GBI Scores (Post- ES) } \\
\hline Response to ESF & \multicolumn{3}{c}{ SOD subtype } \\
\hline & SOD1 & SOD2 & SOD3 \\
No improvement & -19.4 & -31 & -63 \\
Initial response then relapse & 8.3 & 8.3 & -19 \\
Sustained response & 44 & 31 & 29 \\
\hline
\end{tabular}


White British (86\%) with median age 37 years (range 1869). $88 \%$ had undergone prior cholecystectomy. Patients were attributed with the following pre-test diagnoses - 20/ 86 (23\%) SOD1, 53/86 (62\%) SOD2, 13/86 (15\%) SOD3. Median post-ERCP follow up was 12 months (range 2-27). In patients who underwent endotherapy and completed questionnaires: 93\% (14/15) SOD1, 76\% (36/48) SOD2 and $83 \%(10 / 12)$ SOD3 subjectively reported pain improvement post-ERCP (within median 1 month). Sustained response (median 6 months) was noted in 60,30 and $46 \%$ of SOD $1 / 2 / 3$ respectively. Median total GBI scores in the patients who had sustained improvement were +44 (SOD1), +31 (SOD2) and +29 (SOD3). There was a clear correlation between subjective response to ES and GBI scores (see table). Negative total scores were recorded across all SOD subtypes in patients who had no symptom improvement whatsoever following ES. Total GBI scores in all categories were higher in SOD1 than SOD2 than SOD3.

Conclusion ES for SOD1 appears to provide sustained benefit in symptoms and QOL above that achieved in SOD2/3. QOL measured by GBI is strongly correlated to symptomatic response to ES despite pain response being only a minor contributor to the GBI rating. Therefore, GBI may be helpful to determine clinical, emotional and social factors that could help to predict those patients who will respond to ES.

Disclosure of Interest None Declared.

OC-069 MESALAZINE FOR TREATMENT OF DIARRHOEA-PREDOMINANT IRRITABLE BOWEL SYNDROME (IBS-D): A MULTI-CENTRE, PARALLEL GROUP, RANDOMISED PLACEBO CONTROLLED TRIAL

${ }^{1} \mathrm{C}$ Lam*, ${ }^{2} \mathrm{~W}$ Tan, ${ }^{2} \mathrm{M}$ Leighton, ${ }^{3} \mathrm{~J}$ Williams, ${ }^{4} \mathrm{~A}$ Agrawal, ${ }^{5} \mathrm{~S}$ Sen, ${ }^{6} \mathrm{~S}$ Foley, ${ }^{7} \mathrm{M}$ Rutter, ${ }^{8} \mathrm{~A}$ Ramadas, ${ }^{9} \mathrm{P}$ Whorwell, ${ }^{2} \mathrm{~A}$ Montgomery, ${ }^{1} \mathrm{R}$ Spiller. ${ }^{1} \mathrm{~N} I H R$ Nottingham Digestive Diseases Biomedical Research Unit, Nottingham, UK; ${ }^{2}$ University of Nottingham, Nottingham, UK; ${ }^{3}$ Royal Derby Hospital, Derby, UK; ${ }^{4}$ Doncaster Royal Infirmary, Doncaster, UK; ${ }^{5}$ University Hospital of North Staffordshire NHS Trust, Stoke-on-Trent, UK; ${ }^{6}$ Kings Mill Hospital, Sutton in Ashfield, UK; ${ }^{7}$ University Hospital of North Tees, Cleveland, UK; ${ }^{8}$ James Cook Hospital, Middlesbrough, UK; ${ }^{9}$ Wythenshawe Hospital, Manchester, UK

10.1136/gutjnl-2014-307263.69
Introduction "Immune activation" has been described in the mucosa of IBS-D patients which Mesalazine (M) could suppress. Our main aim was to compare the effect of $\mathrm{M}$ versus placebo (P) on stool frequency. Secondary endpoints were abdominal pain, stool consistency and satisfactory relief of IBS symptoms.

Methods All patients were required to have daily stool frequency of $\geq 3 /$ day for more than 2 days/week for 2 weeks and stool consistency of $\geq 25 \%$ type $5-7$ and $\leq 25 \%$ type $1-2$ according to the Bristol Stool Form Scale. Subjects were randomised to either $2 \mathrm{~g} \mathrm{M} / \mathrm{P}$ for a week, to increase to $2 \mathrm{~g}$ twice/day for remaining 11 weeks if tolerated. All participants completed a 12-week stool diary. Since we expected $M$ would require $>2$ months exert its effect, all primary and secondary outcomes were based on the stool diary completed during week 11-12. Satisfactory relief of IBS symptoms was defined as answering 'yes' to weeks 11 and 12 of the stool diary

Results 136 subjects with IBS-D, meeting the Rome III criteria, were randomised to the 2 groups. Mean (SD) age was 47.1 (13.5) years in $\mathrm{P}$ and 42.6 (15.2). Treatment compliance for $\mathrm{P}$ and $\mathrm{M}$ were similar, 59\% and 58\% respectively. Analysis by intention to treat showed $\mathrm{M}$ did not improve bowel frequency, abdominal pain and stool consistency compared to $\mathrm{P}$ during week 11-12. Treatment did not affect satisfactory relief of IBS symptoms, HAD, PHQ15 and EQ-5D VAS scores compared to P. See table below for results.

Conclusion This study did not show any clinically meaningful benefit or harm of $\mathrm{M}$ compared to $\mathrm{P}$ in this group of IBS-D patients. We need better phenotyping/ biomarkers for IBS patients to allow targeting of effective treatments.

Disclosure of Interest C. Lam: None Declared, W. Tan: None Declared, M. Leighton: None Declared, J. Williams: None Declared, A. Agrawal: None Declared, S. Sen: None Declared, S. Foley: None Declared, M. Rutter: None Declared, A. Ramadas: None Declared, P. Whorwell Grant/research support from: Danone, Almirall, Shire, Boehringer Ingelheim UK, Sucampo, Saliz, Chr-Hansen and Norgine, Consultant for: Danone, Almirall, Shire, Boehringer Ingelheim UK, Sucampo, Saliz, Chr-Hansen and Norgine, A. Montgomery: None Declared, R. Spiller Grant/research support from: Lessaffre, Ironwood, Consultant for: Almirall, Astellas, Danone and Sanofi, Conflict with: free drug for clinical trial from Norgine.

\begin{tabular}{|c|c|c|c|c|c|c|}
\hline Mean (SD) & $\begin{array}{l}\text { Baseline } \\
P(n=58)\end{array}$ & $\begin{array}{l}\text { Weeks } 11-12 \\
P(n=58)\end{array}$ & $\begin{array}{l}\text { Baseline } \\
M(n=57)\end{array}$ & $\begin{array}{l}\text { Weeks } 11-12 \\
M(n=57)\end{array}$ & $\begin{array}{l}\text { Between group difference } \\
\text { at week } 11-12^{*}(95 \% \mathrm{Cl})\end{array}$ & $P$ value \\
\hline $\begin{array}{l}\text { Daily mean } \\
\text { stool frequency }\end{array}$ & $3.6(1.8)$ & $2.7(1.9)$ & $3.6(1.6)$ & $2.8(1.2)$ & $\begin{array}{l}0.1 \\
(-0.33,0.53)\end{array}$ & 0.658 \\
\hline $\begin{array}{l}\text { Daily mean } \\
\text { abdominal pain (0-10) }\end{array}$ & $3.6(2.0)$ & $2.2(2.1)$ & $4.1(2.2)$ & $2.8(2.1)$ & $\begin{array}{l}0.07 \\
(-0.54,0.68)\end{array}$ & 0.828 \\
\hline Mean stool consistency & $5.6(1.0)$ & $4.7(1.1)$ & $5.4(0.7)$ & $4.7(1.0)$ & $\begin{array}{l}0.13 \\
(-0.21,0.48)\end{array}$ & 0.452 \\
\hline No. of patient had satisfactory relief of IBS symptoms & 0 & 24 & 0 & 25 & $1.13 * *(0.51,2.47)$ & 0.762 \\
\hline HADS & $8.6(4.3)$ & $6.9(3.6)$ & $9.0(4.5)$ & $7.5(5.0)$ & $\begin{array}{l}0.67 \\
(-0.38,1.72)\end{array}$ & 0.210 \\
\hline PHQ15 & $13.1(5.6)$ & $9.4(5.0)$ & $12.6(5.2)$ & $10.0(5.2)$ & $\begin{array}{l}0.63 \\
(-0.93,2.20)\end{array}$ & 0.428 \\
\hline EQ-5D VAS score & $64.3(20.2)$ & $69.7(18.3)$ & $64.2(20.6)$ & $72.6(19.2)$ & $\begin{array}{l}2.39 \\
(-3.24,8.02)\end{array}$ & 0.406 \\
\hline
\end{tabular}

